\title{
Hydrogel-basierter plasmonischer Ethanolsensor mit schnellen Ansprechzeiten
}

\author{
Christoph Kroh ${ }^{1}$, Roland Wuchrer ${ }^{2}$, Nadja Steinke ${ }^{2}$, Margarita Günther ${ }^{1}$, Gerald Gerlach ${ }^{1}$, Thomas Härtling ${ }^{1,2}$ \\ ${ }^{1}$ Institut für Festkörperelektronik, TU Dresden, Dresden, Deutschland \\ ${ }^{2}$ Fraunhofer Institut für Keramische Technologien und Systeme IKTS, Dresden, Deutschland \\ Kontakt: christoph.kroh@tu-dresden.de
}

\section{Einleitung}

Der Bedarf an effizienten und kostengünstigen Sensoren zur Erfassung chemischer Parameter in Flüssigkeiten steigt in vielen verschiedenen Anwendungsbereichen wie der medizinischen Versorgung oder der Lebensmittelindustrie stetig an. In bspw. Brauereien oder Brennereien ist hierbei insbesondere der Ethanolgehalt ist ein wichtiger Parameter in der Prozessüberwachung.

Gegenwärtige Nachweismethoden für Ethanol basieren auf der indirekten Berechnung des Ethanolgehalts mit Oszillationsdichtemessgeräten [1] oder auf einer spektralen Bestimmung mittels Infrarotspektroskopie [2,3]. Solche Systeme erfordern eine Laborumgebung und sind zeit- und kostenintensiv, wodurch sie für viele kleine und mittlere Brauereien unerschwinglich sind. Im Vergleich dazu haben mobile Laborgeräte wie Hydrometer eine schlechte Auflösung und eine starke Temperaturabhängigkeit [4], weshalb sie sich nicht für eine genaue Überwachung des Ethanolgehalts über einen längeren Zeitraum eignen.

Ein Ansatz zur Lösung dieser Probleme basiert auf der Verwendung von ethanolempfindlichen Hydrogelen. Hydrogele sind hydrophile, unlösliche und vernetzte Polymere, die eine reversible Volumenänderung als Reaktion auf einen chemischen Parameter in ihrer Umgebung zeigen. Durch die Abfrage des Quell- bzw. Entquellzustands des Hydrogels kann somit auf den bspw. die Ethanolkonzentration in der Lösung geschlossen werden. Die Volumenänderung kann beispielsweise mit Hilfe eines Drucksensors [5-8] erfasst werden, bei dem Hydrogelschichten im Bereich von 50 bis $330 \mu \mathrm{m}$ erforderlich sind [5-7]. Aufgrund der Abhängigkeit zwischen Schichtdicke und Ansprechverhalten [9] wird der Gleichgewichtszustand in diesen Systemen typischerweise erst nach mehr als 20 min erreicht $[5,6,8]$. Eine Möglichkeit, die Reaktionszeit von Sensoren auf Hydrogelbasis zu verbessern, besteht darin, den ethanolinduzierten Quellzustand optisch abzufragen. Zu diesem Zweck kombinieren wir ein ethanolempfindliches Hydrogel mit einem nanostrukturierten Goldsensorsubstrat [10]. Die nanostrukturierte Metalloberfläche unterstützt lokalisierte Plasmonenoszillationen [11,12], die mit einem einfachen Transmissionsaufbau angeregt werden können. Die Plasmonenoszillationen sind empfindlich gegenüber Brechungsindexänderungen in der Nähe des Metallsubstrats, die wiederum als spektrale Änderungen des Transmissionsspektrums ausgelesen werden können $[13,14]$.
Während des Quellens und Entquellens des Hydrogels, induziert durch die Änderung der Ethanolkonzentration, diffundiert Wasser in das Gel hinein oder aus diesem heraus, wodurch das Polymer-Wasser-Verhältnis beeinflusst wird. Dies führt zu einer Änderung des effektiven Brechungsindex an der Metall-PolymerGrenzfläche und schließlich zu spektralen Änderungen des Transmissionsspektrums des Sensorsubstrats. Die Empfindlichkeit der Substrate ist auf wenige $100 \mathrm{~nm}$ über der Oberfläche beschränkt, wodurch die Verwendung von sehr dünnen Hydrogelschichten ermöglicht wird. Dadurch kann die Diffusionszeit des Analyten in die Hydrogelschicht verkürzt werden und folglich eine schnellere Reaktionszeit im Vergleich zu anderen auf hydrogelbasierten Sensorsystemen ermöglicht werden.

In dieser Arbeit wird die Kombination eines ethanolempfindlichen Hydrogels auf PolyacrylamidBisacrylamid-Basis mit einem nanostrukturierten Goldsensorsubstrat vorgestellt. Das übergeordnete Ziel dieser Arbeit ist die Entwicklung eines robusten Sensors auf Hydrogelbasis für die Inline-Messung von Ethanol mit einer Lebensdauer von mehr als 10 Tagen.

Es werden das nanostrukturierte Sensorsubstrat, deren Brechzahlsensitivität und der optischen Nachweis der ethanolinduzierten Hydrogelquellung präsentiert. Darüber hinaus werden erste Ergebnisse zur Herstellung dünner Hydrogelschichten und die damit verbundene Verringerung der Sensoransprechzeit dargestellt. Abschließend werden Ergebnisse zu ersten Tests der Anwendung des Messprinzips in alkoholischen Getränken vorgestellt.

\section{Methoden und Materialien}

\section{Plasmonisches Sensorsubstrat und Sensoraufbau}

Die Sensorsubstrate bestehen aus einem $3 \times 3 \mathrm{~mm}^{2}$ großen Nanosäulenfeld in hexagonaler Anordnung, welche mittels Nanoimprint-Lithographie mit dem Mikrokontaktdrucker $\mu \mathrm{CP} \quad 4.1 \quad$ (GeSiM $\mathrm{mbH}$, Großerkmannsdorf, Deutschland) hergestellt wurden. Cycloolefinpolymerfolie (COP) mit einer Dicke von $188 \mu \mathrm{m}$ wurde als Substratträger verwendet. Die Nanosäulen haben einen Durchmesser von ca. $230 \mathrm{~nm}$, einen Abstand von ca. $450 \mathrm{~nm}$ und eine Höhe von ca. $150 \mathrm{~nm}$. Nach der Herstellung wurden die Substrate mit einer $2 \mathrm{~nm}$ dicken Chromschicht und einer $20 \mathrm{~nm}$ dicken Goldschicht beschichtet. Die Sensorsubstrate unterstützen lokalisierte Oberflächenplasmonenoszillationen [11-13], welche bei normalem Lichteinfall in der nanostrukturierten 
Metallschicht angeregt werden können. Aufgrund von Absorptions- und Streueffekten kommt es zu einem lokalen Minimum im Transmissionsspektrum des Sensorsubstrats [13,14]. Diese Position dieses Minimums repräsentiert die Plasmonenresonanzwellenlänge, welche aus einer Überlagerung des einfallenden LED-Spektrums und des Extinktionsspektrums der Nanostruktur resultiert. Die Resonanzwellenlänge verschiebt sich linear mit dem oberflächennahen Brechungsindex (sensitiv bis einige 100 $\mathrm{nm}$ über der Substratoberfläche) der Sensoroberfläche und dient als Sensorsignal [14-17]. Folglich können solche Substrate als Sensorelemente zur Überwachung von Brechungsindexänderungen und ferner als Wandlerelement für Hydrogele verwendet werden. Zur Überwachung der Wellenlängenverschiebung wird ein Transmissionsaufbau verwendet (Abb. 1).

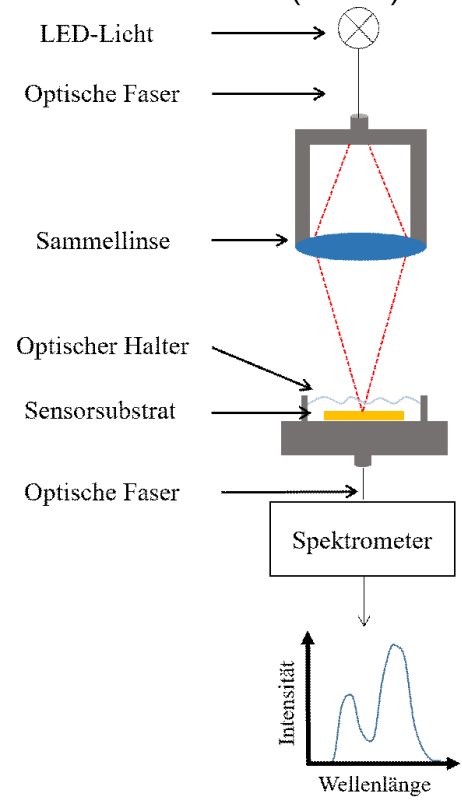

Abb. 1: Schematische Darstellung des Transmissionsaufbaus zur Vermessung der Sensorsubstrate.

Die Substrate werden an einem optischen Halter befestigt und im Spektralfenster von 750-1000 nm mit LED-Licht bestrahlt. Die Transmissionsspektren der Sensorsubstrate werden mithilfe eines Spektrometers (iHR550, Horiba Jobin Yvon GmbH, Unterhaching, Deutschland, Auflösung 0,03 nm) aufgenommen. Die Resonanzwellenlänge wird schließlich aus einer Gaußschen Anpassung jedes Spektrums berechnet. Die mit verschiedenen Wasser-Ethylen-Glykol-Gemischen bestimmte Empfindlichkeit des Sensorsubstrats wurde zu ca. $287 \mathrm{~nm}$ pro Brechungsindexeinheit berechnet (Abb.2).

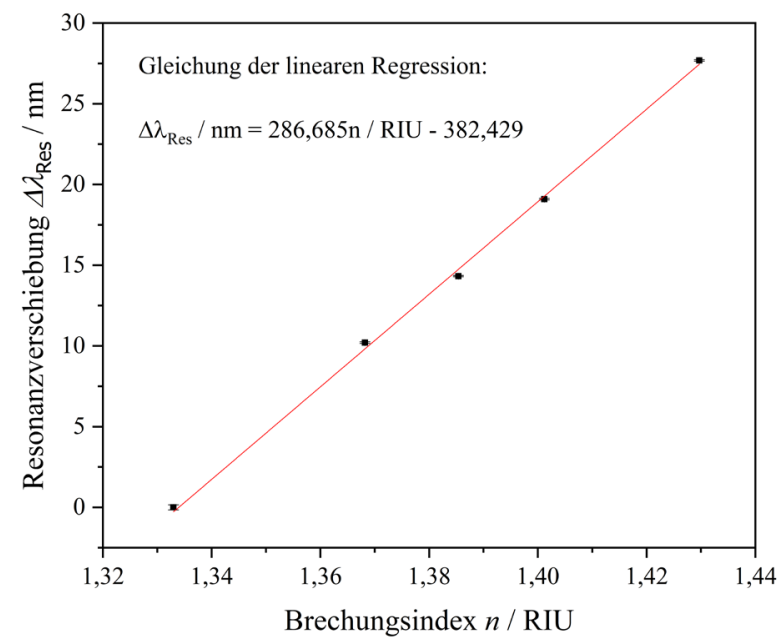

Abb. 2: Brechzahlsensitivität der plasmonischen Sensorsubstrate.

\section{Hydrogelsynthese und -strukturierung}

Zur Reinigung und Aktivierung der Oberfläche wurden die Sensorsubstrate mit Ethanol gespült und $20 \mathrm{~min}$ mit UV I Ozon behandelt. Anschließend wurden die Sensorsubstrate für eine kovalente Hydrogelbindung mit Bisacryloylcystamin (BAC, 0,4 mmol) gelöst in Dimethylsulfidoxid (DMSO) funktionalisiert. Folgend wurden die Hydrogele gemäß [8] synthetisiert. Zur Polymerisation wurde der Photoinitiator Lithiumphenyl2,4,6-trimethylbenzoylphosphinat (LAP, 1 Vol .-\%, 32 $\mu \mathrm{mol})$ zugegeben und unter Stickstoff vermischt. Anschließend wurde $1 \mu \mathrm{l}$ der Stammlösung auf dem Sensorsubstrat abgeschieden. Mithilfe des Mikrokontaktprinters $\mu \mathrm{CP} 4.1$ wurde ein mit Parylene-C beschichteter mikrostrukturierter PolydimethylsiloxanStempel (PDMS) für 10 Minuten in die Lösung gedrückt. Die strukturierten Hydrogele wurden schließlich unter UVLicht (Wellenlängenbereich $320-500 \mathrm{~nm}$ ) für 2 min polymerisiert. Abschließend wurden die Sensorsubstrate gewaschen und $72 \mathrm{~h}$ in destilliertem Wasser und Ethanol konditioniert. Die Dicke der verwendeten Hydrogelschicht wurde zu etwa $4 \mu \mathrm{m}$ bestimmt. Abb. 3 zeigt ein Bild des hydrogelbeschichteten Sensorsubstrats und der Mikrostruktur des Hydrogels.

a)

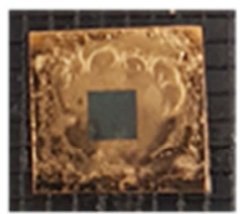

b)

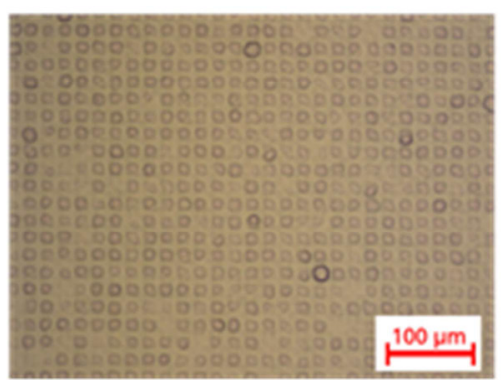


Abb. 3: a) Hydrogel -beschichtetes plasmonisches Sensorsubstrat und b) Mikroskopische Aufnahme der Hydrogel-Struktur.

\section{Ergebnisse}

\section{Optische Überwachung von Ethanolkonzentrationen}

Das reversible Quellen von ethanolempfindlichen Hydrogelen auf Polyacrylamidbasis ist ein bekannter Effekt, der in verschiedenen früheren Arbeiten beschrieben wurde $[18,19]$. Mit zunehmender Ethanolkonzentration entquillt das Hydrogel. Diese reversible Quellung ist reproduzierbar und kann über zahlreiche Zyklen wiederholt werden. Um verschiedene Hydrogelquellzustände zu realisieren, wurden die Sensorsubstrate mit PDMS auf dem optischen Halter befestigt und mit Wasser-Ethanol-Lösungen in unterschiedlichen Konzentrationen bedeckt. Nach einer 15-minütigen Exposition in jeder Lösung wurden die Transmissionsspektren des Sensorsubstrats fünffach aufgenommen und gemittelt. Die Resonanzwellenlänge des jeweiligen Spektrums wurde mit einer Gaußschen Anpassung bestimmt und ihre gemittelte Position über dem jeweiligen Ethanolgehalt aufgetragen. Der Quellgrad des Hydrogels wurde zwischen 0 und 100 vol\% gemessen. Abb. 4 zeigt die Änderung der Resonanzwellenlänge mit zunehmender Ethanolkonzentration. Die dargestellten Messunsicherheiten entstammen der Messunsicherheit der Gaußschen Anpassung und der Standardabweichung der fünffachen Mittelung.

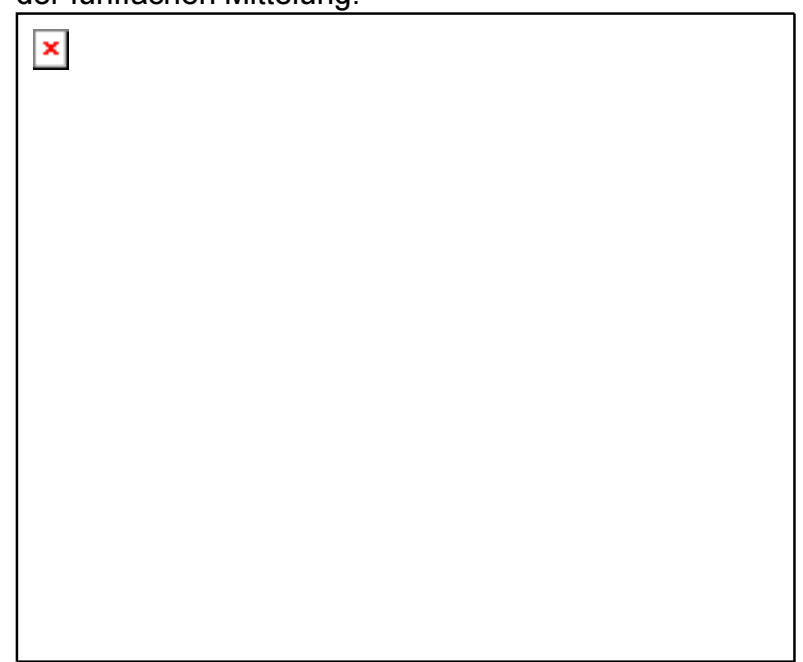

Abb. 4: Resonanzverschiebung bei

Ethanolkonzentrationen bis $100 \mathrm{vol} \%$.

$\begin{array}{cccr}\text { Es ist eine deutliche } & \text { Verschiebung der } \\ \text { Resonanzwellenlänge } & \text { mit } & \text { steigender }\end{array}$ Ethanolkonzentration zu beobachten. Die Steigung der gezeigten Daten wurde mit einer linearen Regression auf $0,1963 \pm 0,013 \mathrm{~nm}$ pro vol\% im unteren Konzentrationsbereich und 0,6832 $\pm 0,037 \mathrm{~nm}$ pro vol\% im oberen Konzentrationsbereich berechnet.

\section{Sensoransprechzeit}

Neben dem Quellverhalten ist ebenso eine schnelle Ansprechzeit eine entscheidende Voraussetzung für den effizienten Einsatz von Sensorelementen. Um die Reaktionszeit zu bestimmen, wurde das Fluidreservoir mit einer Lösung mit 20 vol\% Ethanol gefüllt und die Resonanzwellenlänge über $50 \mathrm{~s}$ gemessen. Anschließend wurde die Lösung wieder auf Wasser gewechselt, um die Reversibilität des Signals zu überprüfen. Abb. 5 zeigt die Daten für die Messung der Sensoransprechzeit. Die dargestellten Unsicherheiten entstammen der Standardabweichung der Gaußschen Anpassung, die zur Bestimmung der Resonanzwellenlänge verwendet wurde.

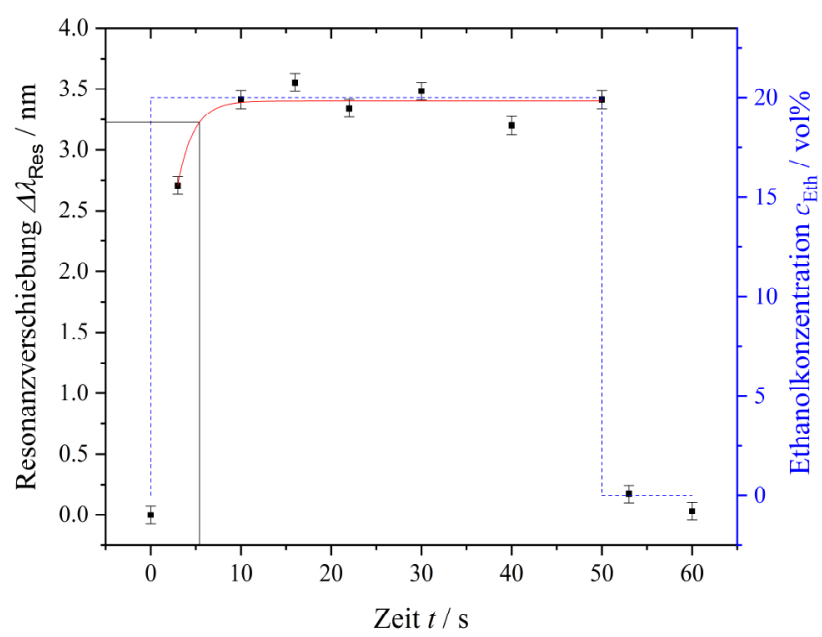

Abb. 5: Ansprechzeit des Sensors bei einem Wechsel von 0 auf 20 vol\% Ethanol.

Die Ergebnisse zeigen eine schnelle und annähernd exponentielle Rotverschiebung der Resonanzwellenlänge. Das Sensorsubstrat reagiert dabei sofort auf die Erhöhung der Ethanolkonzentration und erreicht nach weniger als 10 s $95 \%$ des stationären Wertes, welche als Sensoransprechzeit des Sensors definiert wurde. Nach 50 s wurde die Lösung erneut gegen Wasser ausgetauscht, wodurch die Resonanzwellenlänge in einer ähnlichen Zeitspanne zurück zu ihrer ursprünglichen Position verschiebt. Somit konnte mithilfe der Mikrostrukturierung eine enorme Reduzierung der Reaktionszeit im Vergleich zu anderen hydrogelbasierten Sensorsystemen (mehr als 20 Minuten) [5-8] oder plasmonischen Sensorsubstraten mit nicht strukturierten Hydrogelschichten (mehr als 5 Minuten) [10] erzielt werden.

\section{Messung alkoholischer Getränke}

Um die Anwendung des Messprinzips für industrielle Herstellungsprozesse zu überprüfen, wurde die Überwachung von Ethanolkonzentrationen mittels einer Getränkeprobe von Wodka (40 vol\% Alkohol, Russian Standard Vodka, Russian Standard, St. Petersburg, Russland) getestet. Hierfür wurden unterschiedliche Ethanol-Wasser-Konzentrationen mit den entsprechenden Wodka-Wasser-Gemischen verglichen. Beide Messungen 
wurden mit dem gleichen Sensorsubstrat unter ähnlichen Bedingungen durchgeführt. Darüber hinaus wurde für jede Messung eine fünffache Mittelung der Transmissionsspektren angewendet, um mögliche Messunsicherheiten festzustellen.

Abb. 5 zeigt die Daten dieser Messungen. Sowohl für die Kalibrierungskurve als auch für die Messung der Getränkeprobe ist eine deutliche Rotverschiebung mit nahezu derselben Steigung der Resonanzwellenlänge zu erkennen. Insbesondere bei höheren Ethanol- und Wodka-Konzentrationen stimmen die Daten beider Messungen gut überein. Denn konnten bei geringeren Ethanol- bzw. Wodka-Konzentrationen größere Abweichungen festgestellt werden. Diese können auf Pipettierfehler und unterschiedliches Verdunstungsverhalten der Gemische zurückgeführt werden, welche sich bei geringeren Konzentrationen stärker auf das Messsignal auswirken.

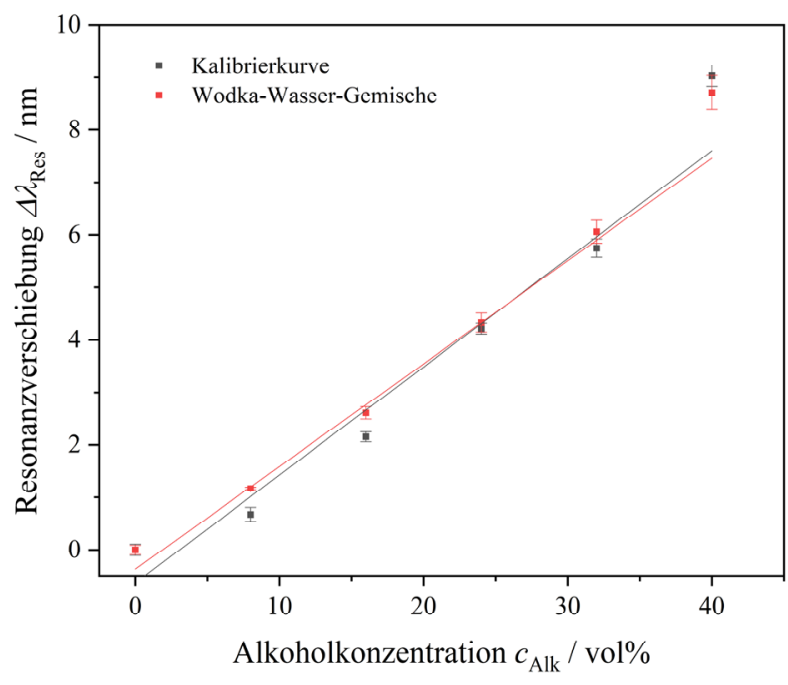

Abb. 5: Vergleich der Verschiebung der Resonanzwellenlänge von Ethanol-Wasser-Gemischen und Wodka-Wasser-Gemischen.

Neben der Wodkaprobe wurde ebenfalls zur Überprüfung der Sensorfunktion in geringen Ethanolkonzentrationen eine Bierprobe (Feldschlößchen Pilsner 5,0 vol\% Alkohol, Feldschlößchen Brauerei, Dresden, Deutschland) vermessen. Hierfür wurden Ethanol-Wasser-Gemische zwischen 0 und 5 vol\% Ethanol mit einer Schrittweite von 1 vol\% und BierWasser-Gemische mit einem Bieranteil von 0 vol\%, 20 vol $\%, 40$ vol $\%, 60$ vol $\%, 80$ vol $\%$ und 100 vol $\%$ auf einem Sensorsubstrat vermessen und verglichen (Abb. 6).

Die Daten zeigen sowohl für die Kalibrierkurve als auch für die Bierproben eine lineare Rotverschiebung der Resonanzwellenlänge. Im Gegensatz zu den Messungen der Wodkaprobe unterscheiden sich die Anstiege der Kurven und somit die Sensitivitäten jedoch stark. Die Sensitivität der Kalibrierkurve wurde zu $(0,1446 \pm 0,008)$ $\mathrm{nm} / \mathrm{vol} \%$ bestimmt. Die Sensitivität der Bierprobe liegt mit $(0,599 \pm 0,036) \mathrm{nm} / \mathrm{vol} \%$ um einen Faktor vier höher.
Diese enormen Unterschiede können anhand der Inhaltsstoffe des Bieres begründet werden. Insbesondere hohe Konzentrationen an Kohlenhydraten und Salzen können sich auf die Funktionsweise des Sensors auswirken und müssen somit ebenfalls überwacht werden.

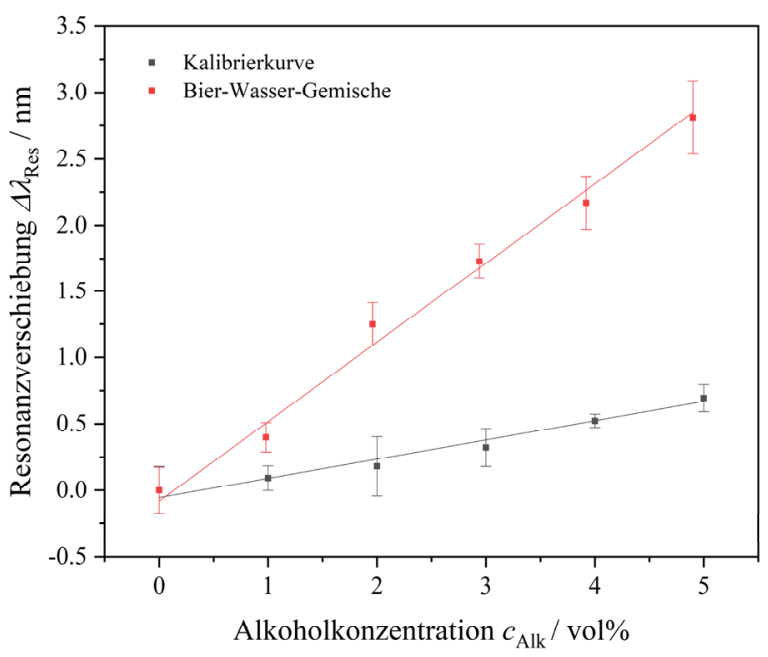

Abb. 5: Vergleich der Verschiebung der Resonanzwellenlänge von Ethanol-Wasser-Gemischen und Bier-Wasser-Gemischen.

\section{Diskussion}

In dieser Arbeit wurde eine optische Nachweismethode vorgestellt, mit der Brechzahlen und Ethanolkonzentrationen in wässrigen Flüssigkeiten nachgewiesen werden können. Hierfür wurde ein nanostrukturiertes plasmonisches Sensorsubstrat mit einem mikrostrukturierten ethanolempfindlichen Hydrogel beschichtet, um die Ethanol-induzierten Brechungsindexänderungen zu bestimmen, die während des Quellens / Entquellens des Hydrogels auftreten. Die Ergebnisse zeigten eine nahezu lineare Rotverschiebung der Resonanzwellenlänge in Ethanolkonzentrationen zwischen 0 und 40 vol\% bzw. 50 und 90 vol\%. Weiterhin konnte mittels der mikrostrukturierten und $4 \mu \mathrm{m}$ dünnen Hydrogelschicht die Reaktionszeit des Sensorsubstrates auf weniger als 10 Sekunden reduziert werden. Dies ermöglicht eine sofortige Erfassung von Änderungen der Ethanolkonzentration auch in sehr dynamischen Prozessen. Abschließend wurde die Anwendbarkeit des Messprinzips für Herstellungsprozesse alkoholischer Getränke mittels einer Wodka- und einer Bierprobe untersucht. Es konnte eine gute Übereinstimmung zwischen der Wodkaprobe und den Kalibrierdaten festgestellt werden. Bei der Vermessung von Bier konnten jedoch aufgrund der Vielzahl an Inhaltsstoffen große Unterschiede in den Sensitivitäten festgestellt werden. Hieraus lässt sich schließen, dass eine genauere Untersuchung der Querempfindlichkeiten des Sensors notwendig ist und ggf. eine Kalibrierung der Substrate auf die zu messende Flüssigkeit vorgenommen werden muss. 
Insgesamt sprechen die hohe Empfindlichkeit und die kurze Reaktionszeit jedoch für die Eignung des Substrats für plasmonische Sensoren als Plattform für die InlineProzessüberwachung in der Lebensmittelindustrie.

Zukünftige Arbeiten werden sich auf die Bestimmung der Nachweisgrenze (LoD) und der Bestimmungsgrenze (LoQ) konzentrieren. Darüber hinaus ist die Kombination des Sensorsubstrates mit einem kompakten und robusten Sensoraufbau notwendig, der aktuell in unserer Gruppe entwickelt wird [20]. Dies ebnet den Weg zu einer kompakten und miniaturisierten Sensoreinheit zur Bestimmung von Flüssigkeitsparametern wie der Ethanolkonzentration.

\section{Literatur}

[1] GONZALEZ-RODRIGUEZ, J.; PEREZ-JUAN, P.; LUQUE DE CASTRO, M. D.: Determination of ethanol in beverages by flow injection, pervaporation and density measurements. Talanta, 2003, Nr. 59, S. 691696.

[2] CAVINTO, A. G.; MAYES, D.M.; GE, Z.; CALLIS, J.B.: Noninvasive Method for Monitoring Ethanol in Fermentation Processes Using Fiber-Optic NearInfrared Spectroscopy. Anal. Chem., 1990, Nr. 62, S. 1977-1982.

[3] MICHEL, K.; BUREAU, B.; BOUSSARD-PLEDEL, C.; JOUAN, T.; ADAM, J.L.; STAUBMANN, K.; BAUMANN, T.: Monitoring of pollutant in waste water by infrared spectroscopy using chalcogenide glass optical fibers. Sens. and Act. B, 2004, Nr. 101, S. 252259.

[4] OSORIO, D.; PEREZ-CORREA, J. R.; AGOSIN, E.; CABRERA, M.: Soft-sensor for on-line estimation of ethanol concentrations in wine stills, J. Food Eng., 2008, Nr. 87, S. 571-577.

[5] GUENTHER, M.; GERLACH, G.; WALLMERSPERGER, T.; AVULA, M. N.; CHO, S. H.; XIE, X.; DEVENER, B. V.; SOLZBACHER, F.; TATHIREDDY, P.; MAGDA, J. J.: Smart Hydrogelbased biochemical microsensor array for medical diagnostics. Adv. Sci. Tech., 2013, Nr. 85, S. 47-52.

[6] GUENTHER, M.; WALLMERSPERGER, T.; GERLACH, G.: Piezoresistive chemical sensors based on functionalized hydrogel. Chemosensors, 2014, Nr. 2, S. $145-170$.

[7] GERLACH, G.; GUENTHER, M.; SUCHANECK, G.; SORBER, J.; ARNDT, K.-F.; RICHTER, A.: Application of sensitive hydrogels in chemical and $\mathrm{pH}$ sensors. Macromol. Symp., 2004, Nr. 210, S. 403-410.

[8] ERFKAMP, J.; GUENTHER, M.; GERLACH, G.: Hydrogel-based piezoresistive sensor for the detection of ethanol. J. Sens. Sens. Syst., 2018, Nr. 7, S. 219226.

[9] TANAKA, T.; FILLMORE, D. J.: Kinetics of swelling of gels. J. Chem. Phys., 1979, Nr. 70, S. 1214-1218.

[10] KROH, C.; WUCHRER, R.; STEINKE, N.; GUENTHER, M.; GERLACH, G.; HÄRTLING, T.: Hydrogel-based plasmonic sensor substrate for the detection of ethanol. Sensors, 2019, Nr. 19, S. 1264 pp.

[11] YANG, S.-C.; HOU, J.-L.; FINN, A.; KUMAR, A.; GE, Y.; FISCHER, W.-J.: Synthesis of multifunctional plasmonic nanopillar array using soft thermal nanoimprint lithography for highly sensitive refractive index sensing. Nanoscale, 2015, Nr. 7, S. 5760-5766.

[12] LEE, K. L.; CHANG, C.-C.; YOU, M.-L.; PAN, M.-Y.; WEI, P.-K.: Enhancing the surface sensitivity of metallic nanostructures using oblique-angle-induced fano resonances. Sci Rep., 2016, Nr. 6, S. 33126 pp.

[13] PILARIK, M.; SÍPOVA, H.; KVASNICKA, P.; GALLER, N.; KRENN, J. R.; HOMOLA, J.: High-resolution biosensor based on localized surface plasmons. Opt. Express, 2012, Nr. 20, S. 672-680.

[14] ZALYUBOVSKIY, J.; BOGDANOVA, M.; DEINEGA, A.; LOZOVIK, Y.; PRIS, A. D.; AN, K. H.; HALL, W. P., POTYRAILO, R. A.: Theoretical limit of localized surface plasmon resonance sensitivity to local refractive index change and its comparison to conventional surface plasmon resonance sensor. J. Opt. Soc. Am., 2012, Nr. 29, S. 994-1002.

[15] DMITRIEV, A.: Nanoplasmonic Sensors. SpringerVerlag, New York, USA, 2012.

[16] SVEDENDAHL, M.; CHEN, S.; DMITRIEV, A.; KALL, $M$.: Refractometric sensing using propagating versus localized surface plasmons: A direct comparison. Nano Lett., 2009, Nr. 9, S. 4428-4433.

[17] MAYER, K. M.; HAFNER, J. H.: Localized surface plasmon resonance sensors. Chem. Rev., 2011, Nr. 111, S. 3828-3857.

[18] CAYKARA, T.; DOGMUS, M.: The effect of solvent composition on swelling and shrinking properties of poly(acrylamideco-itaconic acid) hydrogels. Eur. Polym. J., 2004, Nr. 40, S. 2605-2609.

[19] GUENTHER, M.; GERLACH, G.: Hydrogels for Chemical Sensors. In Hydrogel Sensors and Actuators-Engineering and Technology, SpringerVerlag, Berlin, Heidelberg, Deutschland, S. 165-195, 2009.

[20] WUCHRER, R.; AMREHN, S.; LIU, L.; WAGNER, T.; HÄRTLING, T.: A compact readout platform for spectral-optical sensors. J. Sens. Sens. Syst., 2016, Nr. 5, S. 157-163.

\section{Danksagung}

Die Autoren danken Rüdiger Rosenkranz für die SEMArbeit. Darüber hinaus danken die Autoren Jan Erfkamp für seine Mitarbeit bei der Hydrogelsynthese. Die Förderung durch das DFG-Graduiertenkolleg 1865 und das BMBF-VIP + -Programm unter der Stipendiennummer 03VP01970 ist dankbar. 